\title{
Performance Evaluation of the ARCHITECT i2000 for the Determination of Whole Blood Cyclosporin $\mathrm{A}$ and Tacrolimus
}

\author{
Misun Lim and \\ Yong-Wha Lee \\ Department of Laboratory \\ Medicine and Genetics, \\ Soonchunhyang University \\ Bucheon Hospital, \\ Soonchunhyang University \\ College of Medicine, \\ Bucheon, Korea
}

\footnotetext{
Corresponding author:

Yong-Wha Lee

Department of Laboratory

Medicine and Genetics,

Soonchunhyang University

Bucheon Hospital,

Soonchunhyang University

College of Medicine, 170 Jomaru-

ro, Wonmi-gu, Bucheon 14584,

Korea

Tel: +82-32-621-5943

Fax: +82-32-621-5944

E-mail: lywmd@daum.net

pISSN: 2384-2458

elSSN: 2288-7261
}

\begin{abstract}
Maintaining immunosuppressant concentrations within the therapeutic range in organ recipients requires regular monitoring. The blood concentrations of immunosuppressants are routinely measured using one of several automated immunoassays, such as chemiluminescence immunoassays (CLIAs) and liquid chromatography-tandem mass spectrometry (LC-TMS). The ARCHITECT i2000 immunoassay analyzer (Abbott Diagnostics, USA) was developed as an automated CLIA analyzer for the measurement of cyclosporin A and tacrolimus in whole blood. Here, the precision and linearity of the ARCHITECT i2000 analyzer for the detection of cyclosporin A and tacrolimus in whole blood were evaluated according to Clinical and Laboratory Standards Institute guidelines and were compared with those of an LC-TMS detection method. The total coefficient of variation for the two drugs was less than $10 \%$, and they showed linearity values of 0.97 or more, which was within the manufacturer's range. The measurements of both immunosuppressants by the ARCHITECT i2000 were closely correlated with measurements determined by LC-TMS. However, most measurements were lower with LC-TMS than with the ARCHITECT i2000. Measurement of cyclosporin A and tacrolimus in whole blood using the ARCHITECT i2000 showed very satisfactory performance in terms of precision and linearity as well as good correlation with the comparative method.
\end{abstract}

(J Lab Med Qual Assur 2019;41:117-119)

Key Words: Cyclosporin A, Tacrolimus, Evaluation, ARCHITECT i2000
이식 환자에서 면역억제제 투여 시 치료적 약물농도 구간을 유지하고 독성을 최소화하며 이식 거부위험을 최소화하기 위 해 지속적으로 cyclosporin A와 tacrolimus 등의 면역억제제 에 대한 혈중농도 모니터링이 요구된다. 약물농도의 표준측정 법으로 측정물질에 대한 특이도와 정확도를 높인 액체 크로마 토그래피 질량분석법이 이용될 수 있으나 시간과 비용이 많 이 소요되는 단점이 있다[1]. 근래에 화학발광 면역측정법 원 리가 적용된 자동화 면역분석기기로서 다양한 표지자검사를 수행할 수 있는 ARCHITECT i2000 (Abbott Diagnostics, Abbott Park, IL, USA)가 임상검사실에서 혈중 약물농도에 많이 이용되고 있다[2].

본 연구에서는 Clinical and Laboratory Standards
Institute (CLSI)에 제시된 평가지침에 따라 ARCHITECT $\mathrm{i} 2000$ 을 이용하여 측정한 혈중 cyclosporin A와 tacrolimus 농도에 대하여 정밀도, 직선성과 상관성을 평가하였다[3-5]. Cyclosporin A와 tacrolimus검사가 의뢰된 전혈 검체와 내부 정도관리용 물질을 이용하여 평가하였다. 두 종목에 대해 세 농도의 정도관리용 물질을 이용하여 CLSI 기준에 맞추어 5 일 간 오전 · 오후에 반복검사 수행 후 총 변이계수를 구하였고, 고농도와 저농도의 물질을 이용하여 5 가지 농도로 희석 후 반 복 측정하여 직선성을 보이는 구간을 검증하였으며, 3,600 개 의 다양한 값을 보이는 검체를 이용하여 액체 크로마토그래피 질량분석법이 적용된 Waters Acquity UPLC TQ-Detector (Waters Corp., Milford, MA, USA)와의 상관성을 Passing- 


\section{Journal of LABORATORY MEDICINE and QUALITY ASSURANCE}

\section{Misun Lim et al • ARCHITECT i2000 for Cyclosporin A and Tacrolimus}

Bablok 회귀식을 이용하여 평가하였다.

각각의 평가에 대한 통계는 Microsoft Office Excel 2013

(Microsoft Corp., Redmond, WA, USA) 프로그램을 이용하

Table 1. Precision of cyclosporin A and tacrolimus measurement by the ARCHITECT i2000 with high-, medium-, and low-level quality control materials (controls 1, 2, and 3)

\begin{tabular}{clccc}
\hline Items (units) & Level & $\begin{array}{c}\text { Mean } \pm- \\
\text { standard } \\
\text { deviation }\end{array}$ & $\begin{array}{c}\text { Coefficient } \\
\text { of variation } \\
(\%)\end{array}$ & $\begin{array}{c}\text { Desirable } \\
\text { precision } \\
(\%)^{*}\end{array}$ \\
\hline $\begin{array}{c}\text { Cyclosporin } \\
\text { A (ng/mL) }\end{array}$ & Low & $78.7 \pm 7.29$ & 9.3 & 15.0 \\
& Medium & $273.5 \pm 19.50$ & 7.4 & \\
Tacrolimus & Low & $722.4 \pm 59.80$ & 8.3 & \\
(ng/mL) & Medium & $3.8 \pm 0.22$ & 5.8 & 10.0 \\
& High & $18.6 \pm 0.59$ & 6.6 & \\
\hline
\end{tabular}

The instrument used was from the following company: ARCHITECT i2000 (Abbott Diagnostics, Abbott Park, IL, USA).

${ }^{*}$ Based on the manufacturer's criteria.

Table 2. Linearity of cyclosporine A and tacrolimus measurement by the ARCHITECT i 2000

\begin{tabular}{lcccc}
\hline \multicolumn{1}{c}{ Items } & Units & $\begin{array}{c}\text { Linearity } \\
\text { range }\end{array}$ & $\boldsymbol{R}^{2}$ & Best fit \\
\hline Cyclosporin A & $\mathrm{ng} / \mathrm{mL}$ & $40-1,500$ & 0.999 & Linear \\
Tacrolimus & $\mathrm{ng} / \mathrm{mL}$ & $3-30$ & 0.994 & Linear \\
\hline
\end{tabular}

The instrument used was from the following company: ARCHITECT i2000 (Abbott Diagnostics, Abbott Park, IL, USA).

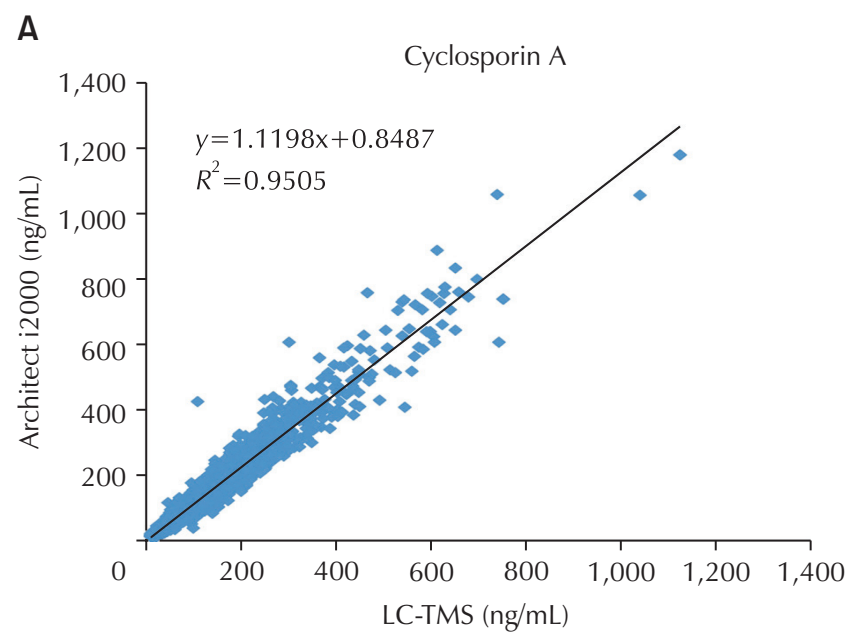

여 분석하였다.

두 종목의 총 변이계수는 고농도물질은 8.0\%-8.3\%의 값을 보였고 저농도물질은 5.8\%-9.3\%의 값을 보였다(Table 1). 직 선성 평가에서 두 종목 모두 제조사 제시범위 내에서 결정계수 가 0.97 이상이었다(Table 2). 액체 크로마토그래피 질량분석 법과의 상관성 평가에서 두 종목 모두 상관계수가 0.9 이상으 로 나타나 밀접한 상관성을 보였으나 액체 크로마토그래피 질 량분석으로 측정된 결과의 대부분이 ARCHITECT i2000으로 측정된 결과보다 cyclosporine $\mathrm{A}$ 와 tacrolimus 각각 평균적으 로 $12.0 \%$ 와 $3.7 \%$ 낮은 것으로 관찰되었다(Figs. 1, 2).

ARCHITECT i2000을 이용한 혈중 cyclosporin A와 tacrolimus 농도 측정은 정밀도와 직선성에 있어서 매우 만족 할 만한 성능을 보였고, 액체 크로마토크래피 질량분석법과 도 좋은 상관성을 보였다. ARCHITECT i2000을 이용한 혈중 cyclosporin $\mathrm{A}$ 와 tacrolimus 농도 측정은 이식 환자에서 지속 적으로 cyclosporin A와 tacrolimus 등의 면역억제제에 대한 신속하고 정확한 혈중농도 모니터링이 가능한 유용한 검사법 으로 평가되었다.

\section{감사의 글}

본 연구에 지원을 아끼지 않은 한국애보트(주)에 깊은 감사를 드린다.
B

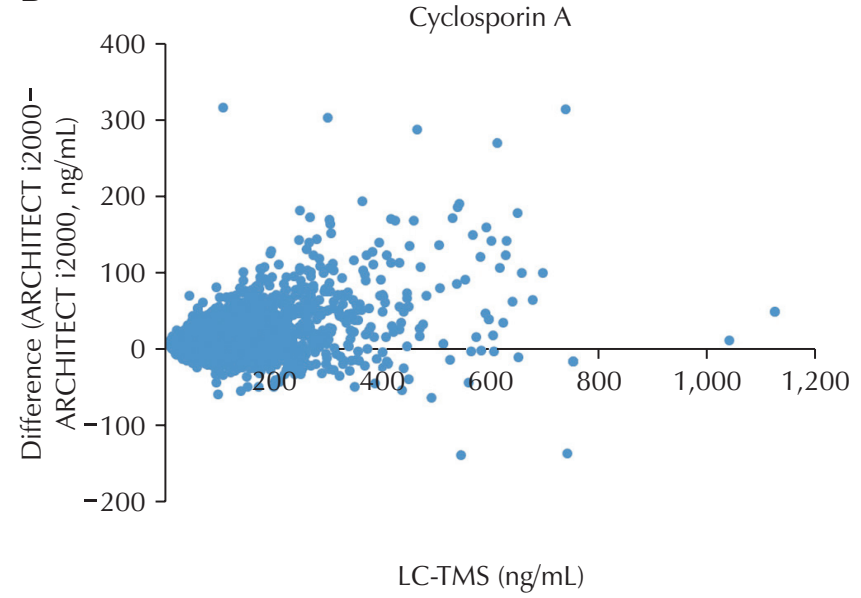

Fig. 1. Comparison of whole blood cyclosporin A measurement by ultra performance liquid chromatography with tandem mass spectrometry and the ARCHITECT i2000, which uses a chemiluminescence immunoassay method. (A) Passing and Bablok regression plot and (B) Bland-Altman plot. The instrument used was from the following company: ARCHITECT i2000 (Abbott Diagnostics, Abbott Park, IL, USA). Abbreviation: LC-TMS, liquid chromatography-tandem mass spectrometry. 
A

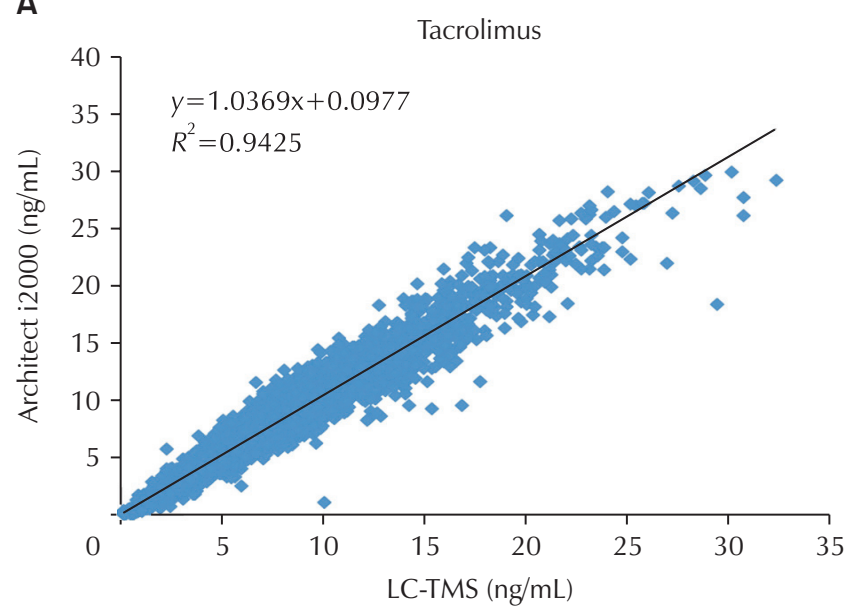

B

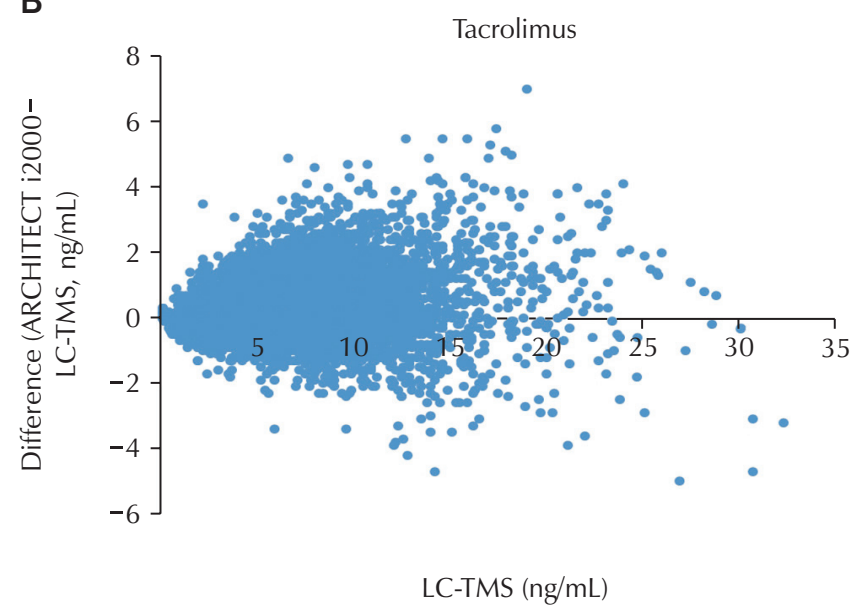

Fig. 2. Comparison of whole blood tacrolimus measurement by ultra performance liquid chromatography with tandem mass spectrometry and the ARCHITECT i2000, which uses a chemiluminescence immunoassay method. (A) Passing and Bablok regression plot and (B) Bland-Altman plot. The instrument used was from the following company: ARCHITECT i2000 (Abbott Diagnostics, Abbott Park, IL, USA). Abbreviation: LC-TMS, liquid chromatography-tandem mass spectrometry.

\section{REFERENCES}

1. Poquette MA, Lensmeyer GL, Doran TC. Effective use of liquid chromatography-mass spectrometry (LC/MS) in the routine clinical laboratory for monitoring sirolimus, tacrolimus, and cyclosporine. Ther Drug Monit 2005;27:144-50.

2. Wu AH. A selected history and future of immunoassay development and applications in clinical chemistry. Clin Chim Acta 2006;369:119-24.

3. Clinical and Laboratory Standard Institute. Evaluation of the precision performance of clinical chemistry devices: approved guideline second edition (EP5-A2). Wayne (PA): Clinical and Laboratory Standard Institute, 2004.

4. Clinical and Laboratory Standard Institute. Evaluation of the linearity of quantitative measurement procedures: a statistical approach; approved guideline (EP6-A). Wayne (PA): Clinical and Laboratory Standard Institute, 2003.

5. Clinical and Laboratory Standard Institute. Method comparison and bias estimation using patient samples: approved guideline second edition (EP9-A2). Wayne (PA): Clinical and Laboratory Standard Institute, 2002. 